\title{
(Des)fazer corpo, (re)fazer teoria: um balanço da produção acadêmica nas ciências humanas e sociais sobre intersexualidade e sua articulação com a produção latino-americana*
}

\author{
Paula Sandrine Machado**
}

\section{Resumo}

A produção acadêmica brasileira sobre intersexualidade no campo da antropologia em particular, mas também nas Ciências Humanas e Sociais, é ainda tímida, embora se possa observar seu crescimento nos últimos anos. De forma geral, os trabalhos tomam a perspectiva feminista como referencial para as análises. Neste artigo, será indicada uma espécie de caminho teórico e também político que pode ser traçado entre as questões feministas, mais especificamente ligadas ao campo dos estudos da ciência, e a intersexualidade. Serão abordados alguns aspectos da recente produção acadêmica latino-americana, nas Ciências Humanas e Sociais sobre intersexualidade, apontando para determinadas particularidades da constituição desse campo nesse contexto. Trata-se, assim, de um breve e não exaustivo balanço sobre essas produções na América Latina e o modo como essa também é uma história que começa a ser escrita, bem como articulada e somada às discussões sobre antropologia, gênero e sexualidade no Brasil.

Palavras-chave: Intersexualidade, Estudos de Gênero $e$ Sexualidade, América Latina.

\footnotetext{
* Recebido para publicação em 20 de março de 2014, aceito em 28 de maio de 2014.

** Professora dos Programas de Pós-Graduação em Antropologia Social e em Psicologia Social e Institucional, na Universidade Federal do Rio Grande do Sul. machadops@gmail.com
} 
(Un)do Body, (Re)do Theory: Taking a Stock on the Academic Production in Humanities and Social Sciences Regarding Intersexuality and Its Articulation with the Latin-American Production

\begin{abstract}
Brazilian academic production regarding intersexuality specially in the anthropology field but also in Humanities and Social sciences is still shy. It is possible to observe a grown in the last years though. Generally these works use the feminist perspective as referential to their analyses. This article will point a kind of theoretical and political path that may be written between feminist issues, specially those connected to the science studies field, and intersexuality. Recent aspects of Latin-American academic production will be approached in Humanities and Social sciences about intersexuality, pointing to some peculiar aspects of this field constitution in this context. It treats about a brief and not exhausting balance about this Latin-American production and the way in witch this is a story that starts to be written as well as articulated and added to the broader discussions about anthropology, gender and sexuality in Brazil.
\end{abstract}

Key Words: Intersexuality, Gender and Sexuality Studies, Latin America. 


\section{Introdução ${ }^{1}$}

Em 2000, Raven Kaldera, escritor e ativista intersex, publicou um texto na revista Fireweed, no qual narrava uma situação por ele vivida durante uma conferência. Durante sua exposição, a conferencista fazia referência à "linha" a ser traçada entre homens e mulheres; a essa suposta evidência, estabilidade e verdade de uma linha que localiza os corpos em um dos lados do binário sexual. Com um delineador, Kaldera traçou no meio do rosto uma linha pontilhada que seguia até o peito. Com uma navalha na mão, encaminhou-se até a conferencista, que então o observava apavorada, e disse: "aqui está a tua linha entre homem e mulher". Estendendo-lhe a navalha, sugeriu: "Agora, faz o que dizes". O texto é finalizado com uma provocação de Kaldera: "Espero que tenha entendido. Espero que finalmente tenha entendido que, quando se traça uma linha, essa passa através da carne sensível de alguém" (Kaldera, 2000).

Kaldera denuncia, assim, a fragilidade dessa "linha" que divide o mundo entre homens e mulheres. Mais do que isso, aponta para o fato de que essa marca divisória opera como uma norma que regula práticas e discursos e que também atua concretamente sobre os corpos sexuados através de intervenções que buscam garantir a dicotomia sexual. A linha, efetivamente, corta corpos. Nesse caso, Kaldera está se referindo, mais especificamente, aos procedimentos médico-cirúrgicos que visam adequar os corpos de pessoas intersex a um padrão de corpo sexuado.

No campo político e dos Direitos Humanos, assim como na maior parte da literatura na área das Ciências Humanas e Sociais, tem-se optado pela utilização do termo "intersex/intersexualidade" para fazer referência "a todas aquelas situações em que o corpo sexuado de um indivíduo varia em relação a um standard de corporalidade feminina ou masculina culturalmente vigente" (Cabral; Benzur, 2005:283-284). Essas variações podem estar

\footnotetext{
1 Algumas das análises apresentadas neste artigo encontram-se em minha tese de doutorado (Machado, 2008).
} 
relacionadas a muitos aspectos e seus marcadores "morais" $e$ "biológicos" foram se modificando ao longo do tempo. Já na esfera médica, passou a ser de uso corrente o termo "Distúrbios ou Anomalias do Desenvolvimento Sexual" (DSDs) em detrimento dos antigos termos "intersex", "hermafroditismo" $e$ "pseudohermafroditismo" (Lee et al., 2006).

Desde o século XIX, a intersexualidade tem aparecido na bibliografia e na prática médica por meio de um duplo registro: como lugar do experimento, das intervenções e das teorizações sobre a diferença sexual (o que caracteriza e o que torna um corpo "biologicamente" masculino ou feminino?); e como o risco encarnado da instabilidade da diferença sexual como norma $e$ como verdade, a qual parte os corpos sexuados em duas possibilidades distintas - trata-se de um corpo de mulher ou, e somente ou, de homem. Esses dois registros são levados a operar conjuntamente. A cada iminência de risco, acionam-se práticas de intervenção normatizadoras e modos de compreensão que tentam recompor a dicotomia sexual como imperativo da natureza. A cada experimento, a cada reiteração do binarismo nos corpos e a cada nova teorização, novas instabilidades.

Os outrora chamados "hermafroditas" desempenharam um papel fundamental nos debates sobre as definições relacionadas à diferença sexual, constituindo uma espécie de "casos paradigmáticos", sobretudo para a medicina do século XIX e do início do século $\mathrm{XX}$, pois permitiriam observar in vivo alguns aspectos envolvendo a atuação específica da "natureza" e da "cultura" na ordenação dessa diferença (Löwy, 2006). Nesse contexto, merece destaque não apenas a preocupação com a origem da "diferença sexual", mas também a descoberta dos hormônios sexuais como promessa de desvelamento da "chave" ou ponto inequívoco para entender tal diferenciação (Oudshoorn, 1994; Wijngaard, 1997, Rohden, 2008). Exatamente por desafiarem a dicotomia sexual como verdade e como norma, os corpos intersex constituíram o palco privilegiado de atuação do regime político e de autoridade imposto pelo saber médico sobre os corpos sexuados (Dorlin, 2005). 
Nesse processo, a medicina - e seu programa patologizador compôs de maneira extensiva a história da intersexualidade no Ocidente (Morland, 2009a) e teve um impacto muito forte em sua constituição como uma "história clínica". Ao mesmo tempo, contou essa história como se ela só encontrasse inteligibilidade, e mesmo materialidade, a partir das definições e dos arquivos médicos. Ao realizar-se essa operação epistemológica, a medicina definiu a intersexualidade como algo que pertencia a seu domínio de saber $e$ prática, eliminando, com isso, outros modos de existência, assim como outras éticas, estéticas e eróticas que a envolvem. ${ }^{2}$

Como resposta à hegemonia médica nesse campo, uma série de autoras feministas, pertencentes a diferentes disciplinas, $e$ em consonância com o nascente ativismo intersex dos anos 1990, passaram a se debruçar criticamente sobre os protocolos médicos, seus pressupostos $e$ efeitos violentos $e$ violadores. A intersexualidade, portanto, ganha lugar no feminismo de uma forma muito particular e, neste artigo, indicarei uma espécie de caminho teórico e também político que pode ser traçado entre as questões feministas, mais especificamente ligadas ao campo dos estudos da ciência, e a intersexualidade. Finalmente, focalizarei alguns aspectos da recente produção acadêmica, latinoamericana, nas Ciências Humanas e Sociais sobre intersexualidade, apontando para determinadas particularidades da constituição desse campo nesse contexto. Não pretendo, contudo, ser exaustiva nesta análise, e sim contribuir com um breve balanço sobre essas produções na América Latina e o modo como essa também é uma história que começa a ser escrita, bem como articulada e somada às discussões mais amplas sobre antropologia, gênero e sexualidade no Brasil.

\footnotetext{
2 Agradeço imensamente a Mauro Cabral pelas instigantes discussões que tivemos em torno da temática deste artigo e por todas as valiosas sugestões feitas ao texto. Particularmente em relação ao ponto das determinações medicas sobre a intersexualidade, devo-lhe essa leitura acerca das operações históricoepistemológicas através das quais a medicina definiu a intersexualidade como algo "próprio". Essa definição, sinaliza Cabral, é algo que não deve ser tomado como um "fato", e sim como um "dado" a ser investigado.
} 
Para essa análise, partirei da perspectiva que Mauro Cabral e eu adotamos em um curso que ministramos em agosto de 2013 em Buenos Aires, Argentina, que se chamou "Al desnudo. Una introducción indiscreta a los estudios sobre intersexualidad". Nesse curso, decidimos que não iríamos falar da "intersexualidade em si". Buscamos dar uma espécie de giro epistemológico e "desnudar" aquelas e aqueles que estudam a intersexualidade. Com isso, propusemo-nos a um exercício desconstrutivo que tomava como objeto não apenas os saberes biomédicos, mas também outros saberes que acabaram se naturalizando como críticos, como o feminismo e a teoria queer. Neste artigo, importa entender como a intersexualidade se torna visível nos estudos sobre gênero e sexualidade nas Ciências Humanas Sociais em geral, e mais especificamente na produção antropológica, bem como o modo como essas questões, escritas $e$ lidas hegemonicamente em inglês, são traduzidas na produção latinoamericana e ganham outros lugares teóricos e políticos ${ }^{3}$.

\section{Regulação biomédica dos corpos sexuados e a crítica feminista: intersexualidades em jogo}

Conforme apontam Cynthia Kraus et al. (2008:8), "um dos efeitos mais persistentes da patologização da intersexualidade é a sua invisibilidade". A maior parte das pessoas nunca ouviu falar em intersexualidade, ainda que no Brasil o tema tenha tido alguma repercussão na mídia, especialmente na forma como é mais comumente veiculado: caracterizado como "hermafroditismo". 4 Mesmo no contexto dos debates feministas, essas são problematizações ainda pouco "visibilizadas".

3 Para uma discussão crítica sobre a hegemonia da produção sobre intersexualidade em inglês, ver a coletânea editada por Mauro Cabral "Interdicciones: escrituras de la intersexualidad en castellano" (Cabral, 2009), especialmente a apresentação escrita pelo autor.

4 Podemos citar, em relação a esse aspecto, a emissão de documentários sobre o tema em canais como GNT e Discovery Channel, bem como novelas e seriados com personagens caracterizados como hermafroditas ou intersex (em 1993, a novela Renascer, de Benedito Ruy Barbosa, exibida pela Rede Globo, contava 
Donna Haraway (2004) destaca duas questões que remetem às relações entre feminismo e intersexualidade. Em primeiro lugar, aponta para a relação entre o manejo médico da intersexualidade e da transexualidade e o surgimento da categoria "gênero". Ela lembra que, em 1963, o termo "identidade de gênero" foi apresentado em um congresso pelo psicanalista Robert Stoller. O trabalho de Stoller estava relacionado ao "Projeto de Pesquisa sobre Identidade de Gênero" do Centro Médico para o Estudo de Intersexuais e Transexuais, da Universidade da Califórnia (UCLA). Desde os anos 50, John Money, da Universidade Johns Hopkins, vinha desenvolvendo o que Haraway (2004:217) descreveu como "a versão interacionista do paradigma de identidade de gênero", a partir da qual emergiu uma perspectiva que combinava programas de pesquisa e programas terapêuticos (psicologia, cirurgia etc.). Em segundo lugar, os apontamentos de Haraway indicam que a intersexualidade está relacionada a uma espécie de contínuo "esquecimento feminista", na medida em que a questão dificilmente figura como estando na origem de um dos fundamentos do próprio feminismo.

Se, de um lado, há esse esquecimento para o qual nos sinaliza Haraway, de outro, há, como já indicado, uma espécie de esquecimento acerca das outras histórias possíveis de serem contadas, que não apenas a biomédica, mesmo nos estudos críticos sobre intersexualidade. Tais histórias começam a ser escritas, mas foram pouco consideradas, como se, de certo modo, os $e$ as que começamos a estudar intersexualidade não rompêssemos totalmente com as narrativas médicas, ou seja, tomássemos a intersexualidade como uma questão médica. Interrogar essa história significa deslocar a medicina como entidade autônoma e única produtora da intersexualidade como

com a personagem Buba, descrita como hermafrodita; Heloísa Buarque de Almeida (USP), por meio de comunicação pessoal, durante o $31^{\circ}$ Encontro Anual da Anpocs, em outubro de 2007, lembrou que um capítulo do seriado "Mulher", também da Rede Globo, teria girado em torno de um caso de intersexualidade. Não se pode esquecer, além disso, da repercussão do caso da judoca Edinanci, também apresentada na mídia como "hermafrodita" (França, 2009). 
objeto e situá-la em um espectro mais amplo de dispositivos sócioculturais e tecnológicos que respondem a demandas por normalização. Significa também refletir sobre os modos como os discursos críticos, assim como as práticas, vindos do ativismo intersex e das feministas que passaram a se debruçar sobre a questão a partir dos anos 90, também construíram as suas versões sobre a intersexualidade e sobre o corpo sexuado (Morland, 2005).

Dito isso, é importante ressaltar que, desde as primeiras intervenções clínico-cirúrgico-psicoterapêuticas aplicadas sobre os corpos intersex até os dias atuais, ocorreram mudanças em diversas esferas, como no que concerne ao contexto políticojurídico, às relações sociais, bem como no que se refere à produção de conhecimento e de ferramentas técnico-científicas (no âmbito da saúde em geral e da medicina em particular). Um exemplo dessas transformações é a emergência nos Estados Unidos, nos anos 1990, da ISNA, primeiro grupo de ativismo intersex, e sua dissolução em 2008, marcando um momento bastante particular do ativismo intersex. Outra dessas transformações é a publicação do "Consenso de Chicago", em 2006, que sugere a utilização, no meio médico, do termo "Desordens/Distúrbios ou Anomalias do Desenvolvimento Sexual" (DSD) no lugar da nomenclatura "Intersex" ou "Estados Intersexuais" (Lee at al., 2006). Existem, assim, hiatos e tensões importantes a serem considerados, não apenas em relação aos profissionais de saúde envolvidos com as decisões $e$ acompanhamento de crianças/jovens intersex, mas também no que diz respeito ao contexto mais amplo que inclui outros atores sociais, como as próprias pessoas intersex (envolvidas ou não com a militância política) e seus familiares.

Nas ciências sociais, não existem muitas referências específicas sobre o tema da intersexualidade, ainda que o mesmo venha sendo cada vez mais contemplado, sobretudo desde os anos 1990, a partir de diferentes recortes, desde as perspectivas histórica, sociológica e antropológica. Deve-se mencionar, evidentemente, a abordagem anterior do tema por Michel Foucault, na publicação do curso "Os Anormais", ministrado nos 
anos 1970 (1974/75) (Foucault, 2001) e no prefácio do livro contendo as memórias de Herculine Barbin (Foucault, 1982), cuja publicação original data de 1978.

Conforme sugere a antropóloga Mariza Corrêa, é possível afirmar que, especificamente na literatura antropológica, apenas contemporaneamente o tema tem sido "descoberto" (Corrêa, 2004a). Vale ressaltar que boa parte dessa produção está relacionada aos estudos de gênero, incluindo desde os estudos feministas da ciência até o interesse atual pela diversidade de gênero.

É em direção ao espaço do hospital - às práticas e definições nele produzidas - que se dirigiram as análises críticas dos tratamentos vigentes e para onde se voltou grande parte dos estudos acadêmicos em relação à intersexualidade (Machado, 2012). Autoras como Anne Fausto-Sterling, Suzanne Kessler, Alice Dreger e Sharon Preves passaram, a partir dos anos 1990, a se dedicar mais intensamente ao tema, lançando questionamentos aos protocolos médicos.

A discussão feminista dá a essas pesquisadoras o arcabouço teórico e político para questionar a própria ideia hegemônica de ciência embutida nesses protocolos, assim como a abordagem heterossexista e desigual presente em orientações e condutas que privilegiavam uma preocupação com a "funcionalidade" da genitália masculina, o que ficava evidente nas primeiras argumentações médicas de que era mais difícil tecnicamente construir um pênis (capaz de penetrar) do que uma vagina (capaz de ser penetrada). O conhecido trocadilho entre os médicos a propósito das cirurgias realizadas em intersexuais: "It is easier to poke a hole than to build a pole" [É mais fácil cavar um buraco do que construir um poste] é uma explicitação desse fato.

lain Morland (2005) aponta que, de forma geral, essas autoras, precursoras nos estudos sobre intersexualidade, utilizaram o tema para explicar "outras coisas". Denunciaram os protocolos médicos como "exemplo de androcentrismo", como exemplo das valorações desiguais entre homens $e$ mulheres que eram sustentadas nos campos médico e biológico. Como dirá Morland, 
mais tarde, essas mesmas feministas se encaminharam lentamente em direção a reformulações no conceito de gênero e a um olhar crítico em relação ao campo dos estudos feministas da ciência, passando a pensar a intersexualidade enquanto "exemplar da injustiça e contestação sexual/política" (Morland, 2005:57), tensionando, assim, a reflexão sobre as possibilidades $e$ impossibilidades das pessoas intersex serem pensadas enquanto sujeitos do feminismo. No entanto, como o autor reforça, a construção da intersexualidade como "exemplo" é uma marca desse campo.

As análises a partir dos estudos queer apontaram para possibilidades analíticas que problematizavam a fixidez das identidades sexuais e questionavam, ainda mais, a ideia de natureza (e da natureza binária da diferenciação sexual). De acordo com Morland (2009b), tais análises corroboraram as críticas aos protocolos médicos, os quais buscavam, a qualquer custo, garantir a estabilidade das dicotomias de sexo e gênero através das intervenções nos corpos intersex. No entanto, Morland também indaga sobre que tipo de críticas à cirurgia genital é possível a partir dos estudos queer. É como se a crítica à cirurgia só funcionasse se sustentada por um ideal de corpo subversivo relacionado à diversidade sexual, possível de ser encarnado pelos corpos intersex não operados. A intersexualidade acaba sendo usada, novamente, como exemplo. Dessa vez, para o desmantelamento do binário sexual. Para Morland, essa perspectiva deixa poucas alternativas para a experiência intersex: ou encarna-se a subversão a uma biologia binária normativa e presume-se, daí, uma experiência de prazer, ou produz-se, em função das manipulações médicas, a vergonha.

É preciso destacar, ainda, que houve uma implicação entre a produção científica emergente nesse campo e o também nascente movimento ético e político intersex nos anos 1990 nos EUA. Por outro lado, há também uma relação histórica entre a própria emergência do ativismo intersex nos Estados Unidos e a academia. A formação da ISNA foi anunciada por Charyl Chase em uma carta em reação à publicação de um artigo de Anne 
Fausto-Sterling intitulado "Os cinco sexos: por que macho e fêmea não são suficientes" [The five sexes: Why male and female are not enough] (Chase, 1998).

É importante ressaltar que os debates em torno da intersexualidade apontam para novas questões, que não estavam no horizonte de algumas das feministas que se propuseram a estudar a ciência a partir da emergência das novas tecnologias reprodutivas. Para essas e outras autoras, o tema passa a se colocar como questão que contribui para a constituição de perspectivas feministas críticas, de onde surge a necessidade (inevitabilidade?) de repensar as categorias sexo e gênero. As interrogações acerca do "sujeito do feminismo" nesse campo levantam reflexões sobre determinadas distribuições diferenciais nas economias epistemológicas e políticas, através das quais a intersexualidade se constrói como objeto de estudo $e$ exemplificação, ao mesmo tempo em que as pessoas intersex não figuram como sujeitos do feminismo.

\section{E a América Latina?}

No contexto latino-americano, podemos destacar uma produção ainda tímida, mas crescente, dos estudos em intersexualidade no campo das Ciências Humanas e Sociais. Cada vez mais, o tema tem ocupado lugar nas discussões em eventos acadêmicos, sobretudo em grupos de trabalho que se propõem a discutir temas relacionados à diversidade sexual, aos direitos humanos $e$ à ciência $e$ às biotecnologias. $\mathrm{O}$ número de teses $e$ dissertações ainda não é expressivo, mas também tem conhecido um aumento na última década.

Mauro Cabral, filósofo e historiador argentino, talvez tenha sido o primeiro a abordar a temática na América Latina. O autor trabalha com intersexualidade, academicamente, desde 2001, e, politicamente, desde meados dos anos 1990. A partir de um recorte filosófico, tem abordado questões ético-políticas em torno da intersexualidade, como aquelas referentes à cidadania sexual $e$ à intersexualidade como subjetividade política, ao manejo 
sociomédico contemporâneo e aos aspectos jurídicos no que tange à intersexualidade (Cabral, 2004, 2006; Cabral, Benzur, 2005; Cabral, Viturro, 2006).

Nas Ciências Sociais no Brasil, especificamente, esses estudos são inaugurados pelo trabalho da antropóloga Mariza Corrêa. O tema da intersexualidade emerge no trabalho da autora no marco das problematizações acerca das convenções sociais que orientam as práticas concretas de intervenções médicas e a produção do conhecimento científico sobre o corpo (Corrêa, 2004a, 2004b, 2007). Para ela, essas convenções e normatizações podem ser tanto médicas, como jurídicas, antropológicas, entre outras. Corrêa (2004a) oferece, assim, análises críticas em torno das intervenções a que são submetidos os corpos intersex e aproxima alguns elementos relativos a essa discussão de outros, que se referem às práticas de mutilação genital em geral e às biotecnologias.

Ao entender que as intervenções levadas a cabo nos corpos intersex podem ser pensadas através da ideia de mutilação, as análises de Mariza Corrêa permitem relativizar o significado de uma prática amparada por um saber biomédico, constituído como verdade amparada e legitimada pela "ciência". Se nas sociedades primitivas existiria um "aprisionamento" no ritual, na nossa sociedade seríamos capturados pelo saber médico, conforme sugere a autora (Corrêa, 2003). Assim, segundo ela, se as diferenças parecem grandes, nos dois casos tratam-se de valores $e$ regras socioculturais, "convenções culturais", às quais se é levado a aderir para que seja possível a inscrição na cultura.

Outros trabalhos irão se somar a esses, e podemos, sem pretender ser exaustivos, citar alguns deles, como o meu próprio (Machado, 2005; 2008), o de Luciana Lavigne (2006), da Argentina, de Eva Alcántara Zavala (2012), do México, todos de orientação antropológica. Na Psicologia, poderíamos destacar o trabalho de Moara de Medeiros Rocha Santos (2006) e o de Shirley Acioly (2007). No campo da Saúde Coletiva, podemos identificar trabalhos como a tese de Ana Karina Canguçú-Campinho (2012) e, muito recentemente, a dissertação de Anacely Guimarães Costa 
(2014). Merece destaque, ainda, o já citado livro "Interdicciones: escrituras de la intersexualidad en castellano", editado por Mauro Cabral (2009), que reúne textos em castelhano, contando em sua quase totalidade com autores e autoras da América Latina.

No contexto dessa discussão, é importante assinalar que todas e todos que começamos a nos dedicar aos estudos sobre a intersexualidade na América Latina iniciamos nosso percurso a partir da leitura da produção norte-americana de teóricas feministas e/ou queer, como Anne Fausto-Sterling, Alice Dreger, Suzanne Kessler, Sharon Preves e Judith Butler. Uma das principais especificidades da nossa produção em relação à norteamericana parece estar relacionada com o fato de que, nos EUA, houve um importante entrelaçamento da academia com o ativismo intersex, sobretudo o da ISNA, enquanto que, na América Latina, esse não foi o caminho observado. A referência de ativismo que temos ainda é muito perpassada pela imagem da ISNA, mesmo após sua dissolução em 2008.

Em relação às famílias e pessoas intersex com as quais tive contato no Brasil, de forma geral havia um desconhecimento quanto às reivindicações ético-políticas do ativismo intersex. É possível dizer, ainda, que a percepção das mesmas a respeito da intersexualidade passava principalmente pelo registro do "problema de saúde" ou do "corpo mal-formado".Seria necessário um maior investimento analítico no que se refere a esse ponto, mas, talvez, identifique-se, no contexto sóciocultural $e$ histórico em que pesquisei (Machado, 2008), um estreito comprometimento entre as definições biomédicas e as descrições disponíveis para os corpos $e$ as experiências das pessoas intersex $e$ suas famílias, ainda que nem sempre tais descrições e definições coincidam e que, efetivamente, a medicina esteja longe de preencher totalmente a experiência dessas pessoas. mas 


\section{Os objetos, as tecnologias e os feminismos: considerações finais}

Não há dúvidas de que pensar sobre a intersexualidade hoje segue exigindo uma problematização dos protocolos médicos e das cirurgias genitais não consentidas. Os estudos sobre intersexualidade demandam certamente uma perspectiva feminista comprometida com a desconstrução do binarismo sexual, da relação linear e dicotômica entre sexo e gênero e com a crítica a procedimentos que reiteram hierarquias sociais e corporais.

Contudo, pensar sobre intersexualidade teórica, ética e politicamente exige, também, como aponta Mauro Cabral (2013) "outro feminismo", capaz de incorporar a biotecnologia em sua perspectiva de análise, de maneira crítica, mas também criativa e irônica. Nesse ponto, pode-se lembrar de que Donna Haraway (2000), no manifesto ciborgue, refere-se à ironia e à blasfêmia como apostas políticas. Segundo ela,

A ironia tem a ver com contradições que não se resolvem ainda que dialeticamente - em totalidades mais amplas: ela tem a ver com a tensão de manter juntas coisas incompatíveis porque todas são necessárias e verdadeiras (Haraway, 2000:39).

O sujeito político do feminismo, nessa perspectiva, interpelado pela intersexualidade e pela diversidade corporal enquanto horizonte ético, precisa ser deslocado. Nesse deslocamento, não se pode desconsiderar as múltiplas produções, de muitas intersexualidades: aquela narrada pela medicina, pelo movimento político, pelo feminismo, pelos estudos queer. Aquelas produzidas e contadas em inglês e aquelas outras, em castelhano ou português. As traduções, como se pode supor, são muitas e correm de um lado a outro nas disputas políticas $e$ epistemológicas. Nesse processo, atualizam-se lugares no jogo de negociações políticas, posicionam-se sujeitos no jogo social $e$ também hierarquizam-se lugares (e idiomas) de fala. 


\section{Referências bibliográficas}

AlCÁNTARA ZAVAlA, Eva. Llamado intersexual/Discursos, prácticas y sujetos en México. Tesis de doctorado en Ciencias Sociales, Universidad Autónoma Metropolitana-Xochimilco, México, 2012.

CABRAL, Mauro. Ciudadanía (trans) sexual. 2004. Disponível em: $<$ http://www.ciudadaniasexual.org/publicaciones/ganadores.htm $>$. Acesso em: 01 jan. 2005.

CABRAL, Mauro. En estado de excepción: intersexualidad e intervenciones sociomédicas. In: CÁCERES, Carlos F. et alii (eds.). Sexualidad, estigma y derechos humanos: desafios para el acceso a la salud en América Latina. Lima, FASPA/UPCH, 2006, pp.69-90.

CABRAL, Mauro (ed.). Interdicciones: escrituras de la intersexualidad en castellano. Córdoba, Anarrés Editorial, 2009, v.1.

CABRAL, Mauro. Comunicação pessoal, 2013.

CABral, Mauro; BenzUR, Gabriel. Cuando digo intersex: un diálogo introductorio a la intersexualidad. Cadernos Pagu (24), Campinas-SP, Núcleo de Estudos de Gênero-Pagu/Unicamp, 2005, pp.283-304.

CABRAL, Mauro; VITURRO, Paula. (Trans)sexual citizenship in contemporary Argentina. In: CURRAH, Paisley; JUANG, Richard M.; MINTER, Shannon Price. Transgender rights. Minneapolis, University of Minnesota Press, 2006, pp.262-273.

CANGUÇÚ-CAmPINHO, Ana Karina Figueira. A construção dialógica da identidade em pessoas intersexuais: o $\mathrm{X}$ e o $\mathrm{Y}$ da questão. Tese de Doutorado em Saúde Pública, Instituto de Saúde Coletiva, Universidade Federal da Bahia, Salvador, 2012.

CHASE, Cheryl. Until five years ago, intersexuals remained silent. In: FEINBERG, Leslie. Trans liberation: beyond pink or blue. Boston, Beacon Press, 1998, pp.88-93.

CORRÊA, Mariza. Fantasias corporais. In: PISCITELLI, Adriana; GREGORI, Maria Filomena; CARRARA, Sérgio (orgs.). Sexualidade e saberes: convenções e fronteiras. Rio de Janeiro, Garamond, 2004a, pp.173-181. 
CORRÊA, Mariza. Não se nasce homem. Trabalho apresentado no encontro "Masculinidades/Feminilidades", nos Encontros Arrábida 2004, Lisboa, setembro de 2004b.

COSTA, Anacely Guimarães. Fé cega, faca amolada: reflexões acerca da assistência médico-cirúrgica à intersexualidade na cidade do Rio de Janeiro. Dissertação de Mestrado em Saúde Coletiva, Universidade do Estado do Rio de Janeiro, Rio de Janeiro, 2014.

DORLIN, Elsa. Sexe, genre et intersexualité: la crise comme régime théorique. Raisons politiques, $\mathrm{n}^{\circ}$ 18, maio 2005, pp.117-137.

FoUCAULT, Michel, O verdadeiro sexo. In: BARBIN, Herculine. O diário de um hermafrodita. Rio de Janeiro: Francisco Alves, 1982. p. 1-9.

FouCAUlT, Michel. Aula de 22 de janeiro de 1975. In: Os anormais. São Paulo, Martins Fontes, 2001, pp.69-100.

FRANÇA, I. L. Ahora, es toda una mujer: un análisis del caso de Edinanci Silva en los medios latinoamericanos. In: Interdicciones: escrituras de la intersexualidad en castellano, vol.1. Córdoba, Anarrés Editorial, 2009.

HARAWAY, Donna. Manifesto ciborgue: ciência, tecnologia e feminismo socialista no final do século XX. In: SILVA, Tomaz Tadeu da (org. e trad.). Antropologia do Ciborgue: as vertigens do pós-humano. Belo Horizonte, Autêntica, 2000, pp.37-129.

HARAWAY, Donna. "Gênero" para um dicionário marxista: a política sexual de uma palavra. Cadernos Pagu (22), Campinas-SP, Núcleo de Estudos de Gênero-Pagu/Unicamp, 2004, pp.201-246.

KALDERA, Raven. Do It on the Dotted Line. Fireweed: A feminist quarterly of writing, politics, art \& culture, $\mathrm{n}^{\circ} 69,2000, \mathrm{pp} .46-50$.

KraUS, Cynthia et alii. Démédicaliser les corps, politiser les identités: convergences des luttes féministes et intersexes. Nouvelles Questions Féministes (NQF) 27(1), 2008, pp.4-15.

LAVIGNE, Luciana. La intersexualidad: un abordaje de las representaciones socioculturales hegemónicas. Tesis de Licenciatura en Ciencias Antropológicas - Orientación Sociocultural, Facultad de Filosofía y Letras Universidad de Buenos Aires, Buenos Aires, 2006. 
LEE, Peter A. et alii. (in collaboration with the participants in the International Consensus Conference on Intersex organized by the Lawson Wilkins Pediatric Endocrine Society and the European Society for Paediatric Endocrinology). Consensus Statement on Management of Intersex Disorders. Pediatrics (118), 2006, pp.e488e500.

LIMA, Shirley Acioly Monteiro de. Intersexo e identidade: história de um corpo reconstruído. 2007. Dissertação de Mestrado em Psicologia Social, Pontifícia Universidade Católica de São Paulo, São Paulo, 2007

LöWY, Ilana. L'emprise du genre: masculinité, fémininité, inegalité. Paris, La Dispute, 2006.

MACHADO, Paula Sandrine. O sexo dos anjos: o olhar sobre a anatomia e a produção do sexo (como se fosse) natural. Cadernos Pagu (24), Campinas-SP, Núcleo de Estudos de Gênero-Pagu/Unicamp, 2005, pp.249-281.

MACHADO, Paula Sandrine. O sexo dos anjos: representações e práticas em torno do gerenciamento sociomédico e cotidiano da intersexualidade. Tese de Doutorado, Programa de Pós-graduação em Antropologia Social, UFRGS, 2008.

MACHADO, Paula Sandrine. Intersexualidade, intersexualidades: notas sobre alguns desafios teóricos, metodológicos e políticos contemporâneos. In: MISKOLCI, Richard; PELÚCIO, Larissa (orgs). Discursos fora da ordem. Sexualidades, saberes e direitos. São Paulo, Annablume, 2012, pp.179-196.

MORLAND, Iain. The injustice of intersex: feminist science studies and the writing of a wrong. In: ANDERSON, Matthew (ed.). Toward a critique of guilt: perspectives from law and the humanities. Studies in Law, Politics and Society, vol. 36, 2005, pp.53-75.

MORLAND, Iain. Introduction: lessons from the octopus. GLQ: A Journal of Lesbian and Gay Studies, vol. 15, n² 2, 2009a, pp.191-197.

MORLAND, Iain. What Can Queer Theory Do for Intersex? GLQ: A Journal of Lesbian and Gay Studies, vol. 15, n 2, 2009b, pp.285-312.

OUDSHOORN, Nelly. Beyond the natural body: an archeology of sex hormones. London/New York, Routledge, 1994. 
158 (Des)fazer corpo, (re)fazer teoria

ROHDEN, Fabíola. O império dos hormônios e a construção da diferença entre os sexos. História, Ciências, Saúde, Manguinhos 15 (supl.), Rio de Janeiro, junho 2008, pp.133-152.

SANTOS, Moara de Medeiros Rocha. Desenvolvimento da identidade de gênero em casos de intersexualidade: contribuições da psicologia. Tese de Doutorado em Psicologia, Instituto de Psicologia, Universidade de Brasília, Brasília, 2006.

WIJNGAARD, Marianne Van Den. Reinventing the sexes: the biomedical construction of feminity and masculinity. Bloomingthon (Ind.), Indiana University Press, 1997. 